\title{
Development of an Eco-Friendly Electrical Discharge Machine (E-EDM) Using TRIZ Approach
}

\author{
V.S. Sreebalaji ${ }^{1}$ and R. Saravanan ${ }^{2}$ \\ ${ }^{1}$ Department of Mechanical Engineering, Einstein College of Engineering, \\ Tirunelveli-12, Tamilnadu, India \\ ${ }^{2}$ Principal, SSK College of Engineering \& Technology, \\ Coimbatore-105, Tamilnadu, India \\ \{V.S.Sreebalaji,R.Saravanan, balajivsv\} arediffmail.com
}

\begin{abstract}
Electrical Discharge Machine (EDM) is one of the non-traditional machining processes. EDM process is based on thermoelectric energy between the work and an electrode. A pulse discharge occurs in a small gap between the work piece and the electrode and removes the unwanted material from the parent metal through melting and vaporization. The electrode and the work piece must have an electrical conductivity in order to generate the spark. Dielectric fluid acts as a spark conductor, concentrating the energy to a very narrow region. There are various types of products can be produced and finished using EDM such as Moulds, Dies, Parts of Aerodynamics, Automotives and Surgical components. This research work reveals how an Eco friendly EDM (E-EDM) can be modeled to replace die electric fluid and introducing ozonised oxygen in to EDM to eliminate harmful effects generated while machining by using dielectric, to make pollution free machining environment through a new design of EEDM using TRIZ (a Russian acronym for Theory of Inventive Problem Solving) approach, since Eco friendly design is the need of the hour.
\end{abstract}

Keywords: EDM, die electric, Ozonised oxygen, model, Eco friendly, TRIZ.

\section{About EDM}

The EDM process can be compared with the conventional cutting process, except that in this case, a suitably shaped tool electrode, with a precision controlled feed movement is employed in place of the cutting tool and the cutting energy is provided by means of short duration electrical impulses. EDM has found ready application in the machining of hard metals or alloys which cannot be machined easily by conventional methods. It thus plays a major role in the machining of dies, tools, etc., made of tungsten carbides, stellites or hard steels. Alloys used in the aeronautics industry, for example, hastalloy, nimonic, etc., could also be machined conveniently by this process. This process has an added advantage of being capable of machining complicated components.

\section{Introduction to TRIZ}

TRIZ is a problem solving method based on logic and data, not intuition, which accelerates the research ability to solve any kinds of problems creatively from day 
today life to emerging Engineering and Technological field. TRIZ also provides repeatability, predictability and reliability due to its structure and algorithmic approach. "TRIZ" is the (Russian acronym for the "Theory of Inventive Problem Solving"). G.S. Altshuller and his colleagues in the former U.S.S.R. developed the method between 1946 and 1985. He discovered that evolution of technical system follows predictable patterns. Indeed, inventiveness and creativity can be taught. TRIZ, an algorithmic methodology, has validated itself by solving thousands of difficult technical problems. It remained underground in the USSR where it was developed in secret by an intellectual elite. With the fall of the Soviet Union, TRIZ has reemerged in the West. Today many fortune 500 companies are successfully applying TRIZ. TRIZ is a rapid, creative approach for solving problems - not just ordinary problems, but also "impossible-to-solve" problems. The word "impossible" really means "impossible by previously known means." Given a good inventive technique, the impossible becomes the possible. The TRIZ approach is included in a rather rigorous step-by-step process, somewhat similar to an algorithm, although not as rigorous as a formal mathematical algorithm. This problem-solving algorithm is called ARIZ (another Russian acronym meaning "Algorithm for the Solution of Inventive Problems").The research has proceeded in several stages during the last sixty years. The three primary findings of this research are as follows:

1. Problems and solutions are repeated across industries and sciences. The classification of the contradictions in each problem predicts the creative solutions to that problem.

2. Patterns of technical evolution are repeated across industries and sciences.

3. Creative innovations use scientific effects outside the field where they were developed.

TRIZ is an international science of creativity that relies on the study of the patterns of problems and solutions, not on the spontaneous and intuitive creativity of individuals or groups. More than four million patents have been analyzed to discover the patterns that predict breakthrough solutions to problems. TRIZ research began with the hypothesis that there are universal principles of creativity that are the basis for creative innovations that advance technology. If these principles could be identified and codified, they could be taught to people to make the process of creativity more predictable. The short version of this is:

Somebody someplace has already solved this problem (or one very similar to it.) Creativity is now finding that solution and adapting it to this particular problem.

USIT is an Americanized TRIZ version. USIT (i.e. 'Unified Structured Inventive Thinking') is one of such simplified methodologies developed in USA. It recommends a simple yet powerful process of problem solving, composed of three stages, i.e. Problem definition, Problem analysis, and Concept generation. In Japan, TRIZ has been introduced and promoted by Nikkei Mechanical Journal, Mitsubishi Research Institute, Sanno Institute of Management, Professor Hatamura's group at the University of Tokyo and in various pioneering Japanese industries. 


\subsection{Essence of TRIZ}

This question must be most basic and important for us to teach/learn TRIZ. There are many important principles and methods in TRIZ, such as:

1. 39 Altshuller's Generic Engineering Parameters.

2. Contradiction Matrix.

3. 40 Principles of Invention.

4. 76 Standard Inventive Solutions.

5. Trends of Evolution of Technical Systems.

6. ARIZ Algorithm etc.,

\subsection{Current Research Trends in EDM Development}

There are two kinds of research trends are carried out by present researchers as for as EDM is concern. One is called Modeling technique and other one is called Novel technique. Modeling technique deals with Mathematical modeling, Artificial Intelligence and Optimization techniques like Regression analysis, Artificial neural network, Genetic algorithm etc., respectively are used to validate the efforts of input parameters on output parameters since EDM is a complicated process of more controllable input parameters like machining depth, tool radius, pulse on time, pulse off time, discharge current, orbital radius, radial step, offset depth and output parameters like Material Removal Rate (MRR) and Surface Quality(SQ). Novel techniques deals how other (unconventional) machining principle such as ultrasonic can be incorporated into EDM to improve efficiency of machining process by get better material removal rate, surface quality. Introducing novel research in EDM has been started during 1996 and continues nowadays. This research work is a revelation to introduce TRIZ in EDM novel research trend.

\subsection{Problem Context of EDM Research}

To Increase the machining efficiency of EDM in terms of improving the Material Removal Rate(MRR) in EDM.

\subsection{Discussion of Problem}

This is an excellent example of a non-trivial (and hence interesting) problem. There is a desirable output/characteristic that we need (in this case material removal rate) that unfortunately appears to be associated with something costly or unwanted (in this case, increase in machining time). In the language of TRIZ we call this a contradiction.

The identification and resolution of contradictions is a key element of the TRIZ problem solving ethos. Inside all interesting problems we invariably find one or more such contradictions. If we can identify these contractions and resolve them we will have devised a high quality solution to problem - not merely a better compromise, but an innovative solution that breaks free from the existing constraints and provides a step-change towards an ideal system. This contradiction can be expressed as a simple graph, which typically takes this form: 


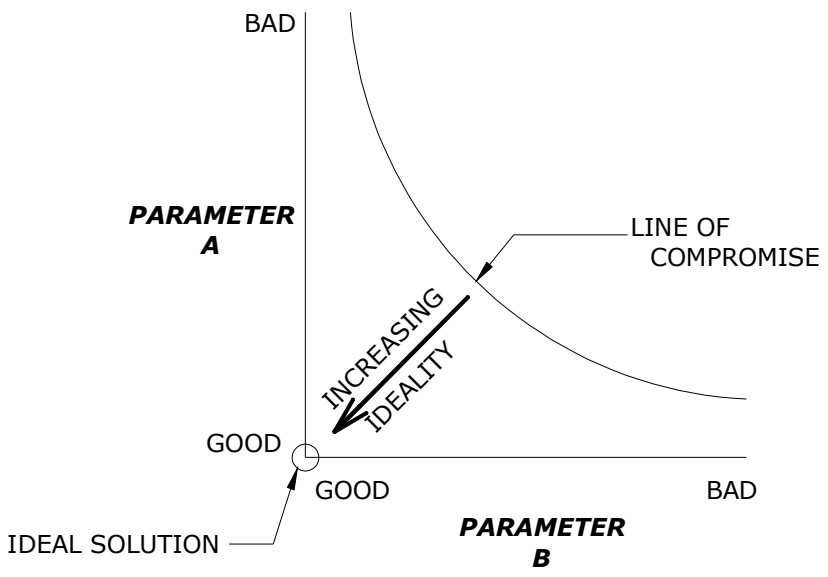

As we try to improve one parameter (material removal rate) the other (machining time) becomes worse, and vice versa. It would appear that we are constrained to remain on line of compromise curve, which defines the set of possible compromise solutions available to us. What we would like to able to do is to move off the line of compromise curve to a more ideal solution - i.e. in the direction shown by the dark arrow. To achieve this we need to find a way of resolving the contradiction.

In the case of this particular problem we are looking for:

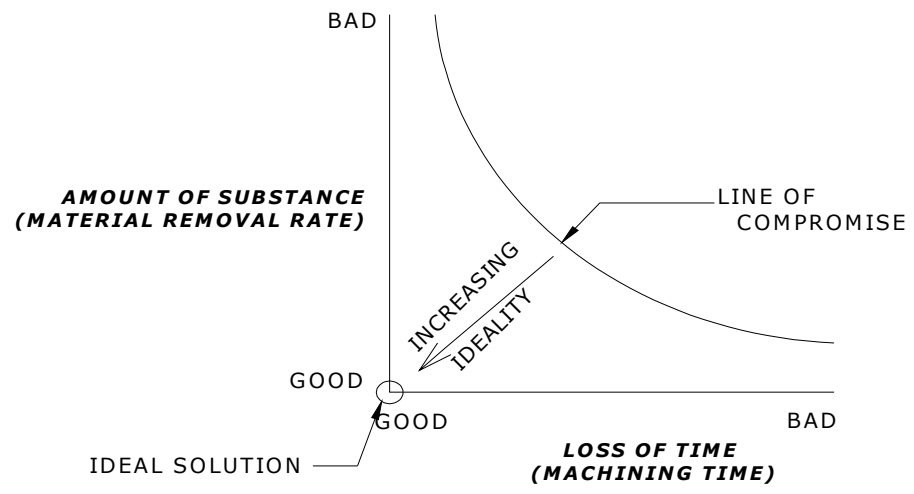

\section{Contradiction to Improve Material Removal Rate (MRR)}

- Improving Parameter: Amount of substance (MRR)

- Worsening Parameter (i.e. Undesired): Loss of time(Increase in Machining time)

These two parameters are used to cross-index the Contradiction Matrix to obtain the following four Inventive Principles that (statistically) have been found to be the most successful ways of obtaining better material removal rate without more machining time. 


\subsection{Suggested Inventive Principles by TRIZ Matrix}

The following inventive principles are suggested by contradiction matrix between the parameters Amount of substance and Loss of time.

- 18 Mechanical vibration

- 38 Strong oxidants

- 35 Parameter Change

- 16 Partial or excessive action

\subsection{Application of Suggested Inventive Principle(s)}

Having derived some suggested Inventive Principles, to apply the principles to this particular problem. This is the part of a recurring theme in TRIZ that the sequence follows a chain like Specific Problem-General Problem - General SolutionSpecialized Solution to Specific Problem.

The next step is, to understand the suggested inventive principles to appreciate the full definition of each principle before attempting to apply it. In this case, the suggested four generic principles' definition with some examples are given below for better reference to understand and to propose a new design.

- Principle 18.Mechanical vibration

- Cause an object to Oscillate or Vibrate.

- Increase its frequency even up to the Ultrasonic,e.g. Distribute powder with vibration.

- Use an object's resonant frequency.

- Use piezoelectric vibrators instead of mechanical ones.

- Use combined ultrasonic and electromagnetic field oscillations.

- Principle 38 .Strong Oxidants

- Replace common air with Oxygen - Enriched air.

- Replace enriched air with pure Oxygen.

- Expose air or oxygen to ionizing radiation.

- Use ionized oxygen.e.g. Ionize air to trap pollutants in an air cleaner

- Replace ozonized (or ionized) oxygen with ozone,.g. Speed up chemical reaction by ionizing the gas before use.

- Principle 16.Partial or Excessive Action (Application of Principle 3 and 9)

- Change an object's structure

- Make each part of an object function in condition most suitable or its operation.

- Make each part of an object fulfill a different and useful function, e.g. Multi function tool.

- Replace with anti- actions to control harmful effects,e.g. Buffer the solution to prevent harm from extreme of $\mathrm{PH}$.

- Create before hand stresses in an object to oppose undesirable working stresses.

- Principle 35.Parameter Change

- Change an object's physical state.

- B. Change the concentration or consistency.

- Change the degree of flexibility.

- Change the temperature. 
At this point in the problem solving process, appropriate problem and technological domain knowledge is important. The final solution derived from these principles were used to model a new design E-EDM and how well this novel approach TRIZ can be used in emerging engineering and technological research domain.

\section{New Design of E-EDM Using TRIZ for Better Material Removal Rate (MRR) in EDM}

The above suggested principles have induced brainstorming to propose a new design.

Principle \# 38 Strong Oxidants suggests replace common air with Oxygen - Enriched air, Replace enriched air with pure Oxygen, Expose air or oxygen to ionizing radiation, Use ionized oxygen, replace ozonized (or ionized) oxygen with ozone. e.g. Speed up chemical reaction by ionizing the gas before use.

As per the principle \# 38 recommendation, we propose a new design E-EDM by introducing ozonized oxygen into EDM by replacing die electric fluid from the existing EDM process, since EDM current research already started introducing dry EDM (i.e. introducing any gas medium) by removing dielectric fluid, it validates our findings that dielectric can be eliminated from the existing design of EDM. As per our team's recommendation that by introducing ozonized oxygen into EDM by eliminating die electric fluid and in further it can speed up material removal rate.

Other recommended principles, Principle \# 18-Mechanical Vibration, Principle \# 35-Parameter Change, Principle \# 16-Partial or Excessive Action, have been used to validate the current EDM research findings in my previous research findings. The proposed new design of EEDM is in the progress of real time design implementation and experimental research.

\section{Conclusion}

This research work reveals how TRIZ has been used in EDM novel research to overcome the limitations of existing EDM process and to a new design of EEDM.TRIZ is an vibrant tool in all means with its powerful search features and offers maximum results with minimal effort. As EDM novel research is concern, works have been started during 1996 and till research continues on improving the machining performance. In this work, using TRIZ, how novelty in EDM research could be achieved with maximum results with minimal effort through a new design of E-EDM. This novel tool can be used to extend in EDM research further.

\section{References}

1. Pandey, P.C., Shan, H.S.: Modern Machining Processes. Tata Mcgraw-Hill Publishing company limited (2003)

2. Abbas, N.M., Solomon, D.G., Bahari, M. F.: A review on current research trends in EDM machining. International Journal of machine tool and manufacture 47, 1214-1228 (2007) 
3. Domb, E., Miller, J., Macgran, E., Slocum, M.: The 39 features of Altshuller's Contradiction matrix

4. Gadd, K.: Andrew Martin of Oxford Creativity. Measurement of acoustic emission in a flying aircraft wing

5. Sreebalaji, V.S., Saravanan, R.: Development of an advance EDM using TRIZ approach. In: Teamtech 2008, International conference at IISc, Bangalore, India (2008) 